\title{
Large Scale Simulation of Dendritic Growth in Pure Undercooled Melt by Phase-field Model
}

\author{
Seong Gyoon KIM, Won Tae KIM, ") Jae Sang LEE, ${ }^{2)}$ Machiko ODE ${ }^{3)}$ and Toshio SUZUKI') \\ RASOM and Department of Materials Science and Engineering, Kunsan National University, Kunsan, 573-360 Korea. \\ 1) RASOM and Department of Physics, Cheongju University, Cheongju, 360-764 Korea. \\ 2) Department of Metallurgy, School of Engineering, the University of Tokyo, Tokyo, 113-8356 Japan. \\ 3) Graduate School, the University of Tokyo, Tokyo, 113-8356 Japan.
}

(Received on August 20, 1998; accepted in final form on December 10, 1998)

\begin{abstract}
In this study we presented a double-grid method for efficient computation of complex dendritic pattern evolving in a large scale solidifying system of pure undercooled melt. The method, which is based on the large difference in phase-field diffusivity and thermal diffusivity in pure material, enables us to use a large time step. The phase field was calculated adaptively only on the grids within the interfacial region and also the thermal field was calculated only within the thermal boundary layer. The computation showed that the complex dendritic patterns with well-developed tertiary arms can be obtained even in a personal computer with a moderate memory space. The computational efficiency of this method, the competitive growth and coarsening of the secondary arms and the tertiary branching from secondary arms were discussed.
\end{abstract}

KEY WORDS: phase-field model; dendritic growth; double-grid method; computational efficiency; large scale simulation.

\section{Introduction}

Phase-field models are known to be very powerful in describing the complex pattern evolution of the interface between mother and new phases in non-equilibrium state. $^{1-10)}$ The models are efficient especially in numerical treatment because all the governing equations are written as unified ones in the whole space of the system without distinguishing the interface from the mother and new phase, and direct tracking of the interface position is not needed during numerical calculation. Recently several phase-field models have been developed mainly for the solidification of pure materials ${ }^{1-6)}$ and they have been extended to the solidification of binary alloys with a single phase ${ }^{7-9)}$ or two solid phases. ${ }^{10)}$

In spite of powerful ability of phase-field models for computation of the pattern evolution in solidification, they suffer from low computational efficiency and very large computer memory resource: even for a quantitative computation of a single dendrite with a few sidebranches, the computational space should be discritized into about one million meshes, ${ }^{6)}$ and so the computational stability condition in a explicit finite difference scheme can be guaranteed only with a very small time step, or in an explicit finite difference scheme one should deal with extremely large matrix. This drawback of the models is due to that all the parameters in the phasefield equation have been determined to be matched with Gibbs-Thomson equation at a vanishing interface thickness, that is, the sharp interface limit condition. ${ }^{4)}$ The sharp interface limit condition can be written as $2 \hat{\lambda} \ll d_{0},{ }^{11)}$ where $2 \lambda$ is interface thickness and $d_{0}$ is the thermal (in pure materials) or chemical (in alloys) capillary length. Noting that in general $d_{0}$ is a factor of 0.1 $\mathrm{nm}$, the interface thickness in computations is restricted within an atomic scale, independent of the characteristic length of the interface patterns, for example, dendrite tip radius.

Thus the first step toward the improvement of computational efficiency in phase-field model must be in relaxation of the sharp interface limit condition. This kind of work for solidification in pure materials has been done by Karma and Rappel ${ }^{1-13)}$ for the first time. They have shown that one (phase-field mobility) of three parameters in phase-field equation can be determined at a finite interface thickness condition (so called thin interface limit condition) without violating Gibbs-Thomson equation at the limit. Also for phase-field model of alloys, recently, we have shown that at a thin interface limit condition all three parameters in phase-field equation can be matched with the material parameters, not only without violating Gibbs-Thomson equation at the limit, but also with introducing the solute trapping effect in a natural way. ${ }^{14)}$

Even though we could be free from the severe condition $2 \lambda \ll d_{0}$ by using the thin interface phase-field model, the computations of the complex interface pattern evolution in a large scale solidifying system still require a fairly large grid system, as long as the interfacial region should 
be resolved with several grids. Thus the next step to enhance the computational efficiency of phase-field model may be in improvement of numerical technique. Until now most computations of pattern evolutions during solidification of pure materials or alloys however have been done by simple finite difference methods without any time-saving technique in numerical algorithms, except of a few studies using an adaptive grid method. ${ }^{15,16)}$

In this study, we present a double-grid method for computations of complex interface pattern evolution in a large scale solidifying system in pure undercooled melts. (The large scale means that our system is very large compared with the width of the main stem of a single dendrite, not the system size itself.) In this method, which is based on the large difference in phase-field diffusivity and thermal diffusivity in pure materials, a double grids with different grid size are used: a larger grid size for thermal field and a small grid size for the phase field. The grid system with a smaller grid size for phase-field calculation is operated adaptively only on the grids within the interfacial region. In order to see the computational efficiency only from the numerical technique, here we will use the conventional sharp interface phase-field model, instead of the thin interface phase-field model. The model system is an undercooled nickel melt.

\section{Governing Equations and Parameters}

The phase field is defined as $\phi=0$ at liquid, $\phi=1$ at solid and it varies continuously from 0 to 1 at the interfacial region. The time-evolution equations of the phase field $\phi$ and temperature field $T$ can be described by ${ }^{4}$

$$
\begin{aligned}
& \frac{\partial T}{\partial t}=D \nabla^{2} T+\frac{\Delta H}{c_{\mathrm{p}}} h^{\prime}(\phi) \frac{\partial \phi}{\partial t} \\
& \frac{\partial \phi}{\partial t}=M(\theta)\left[\left(\nabla \cdot\left(\varepsilon(\theta)^{2} \nabla \phi\right)+\frac{\partial}{\partial y}\left(\varepsilon(\theta) \varepsilon^{\prime}(\theta) \frac{\partial \phi}{\partial x}\right)\right.\right. \\
& -\frac{\partial}{\partial x}\left(\varepsilon(\theta) \varepsilon^{\prime}(\theta) \frac{\partial \phi}{\partial y}\right)-2 w \phi(1-\phi)(1-2 \phi) \\
& \left.-h^{\prime}(\phi) \frac{\Delta H}{T_{\mathrm{m}}}\left(T-T_{\mathrm{m}}\right)\right]
\end{aligned}
$$

where $\theta$ is the angle between the direction normal to the interface and a reference axis ( $x$-axis in this study), $D$ is the thermal diffusivity, $\Delta H$ is the latent heat per unit volume, $c_{\mathrm{p}}$ is the specific heat, $M$ is the phase-field mobility, $\varepsilon$ and $w$ are parameters associated with the interfacial energy and interface thickness, $T_{\mathrm{m}}$ is the melting temperature and $h(\phi)$ is a function that satisfies $h(0)=0, h(1)=1$ and $h^{\prime}(0)=h^{\prime}(1)=0$. As in Ref. 9), we choose

$$
h(\phi)=\phi^{3}\left(10-15 \phi+6 \phi^{2}\right)
$$

The $M(\theta)$ and $\varepsilon(\theta)$ were assumed to have four-fold anisotropies of

$$
\begin{aligned}
M(\theta) & =M_{0}\left[1+\delta_{M} \cos 4\left(\theta-\theta_{0}\right)\right] \\
\varepsilon(\theta) & =\varepsilon_{0}\left[1+\delta_{\varepsilon} \cos 4\left(\theta-\theta_{0}\right)\right] .
\end{aligned}
$$

where $\delta_{M}$ and $\delta_{\varepsilon}$ are anisotropy constants and $\theta_{0}$ is the interface orientation with the maximum anisotropy. The matched asymptotic analysis in sharp interface limit results in the relationships between $M(\theta), \varepsilon(\theta), w$ and the interface energy $\sigma(\theta)$, interface thickness $2 \lambda(\theta)$, linear interface kinetics coefficient $\mu^{\mathrm{k}}(\theta)^{4)}$ :

$$
\begin{array}{r}
2 \lambda(\theta)=2.2 \sqrt{2} \frac{\varepsilon(\theta)}{\sqrt{w}} \equiv 2 \lambda_{0}\left[1+\delta_{\varepsilon} \cos 4\left(\theta-\theta_{0}\right)\right] \\
\sigma(\theta)=\frac{\varepsilon(\theta) \sqrt{w}}{3 \sqrt{2}} \equiv \sigma_{0}\left[1+\delta_{\varepsilon} \cos 4\left(\theta-\theta_{0}\right)\right] \\
\mu^{\mathrm{k}}(\theta)=\frac{3}{1.1} \frac{M(\theta) \Delta H}{u_{\mathrm{m}}} \hat{\lambda}(\theta) \\
\equiv \mu_{0}^{\mathrm{k}}\left[1+\delta_{M} \cos 4\left(\theta-\theta_{0}\right)\right]\left[1+\delta_{\varepsilon} \cos 4\left(\theta-\theta_{0}\right)\right]
\end{array}
$$

From these relationships we can determine the three parameter $M_{0}, \varepsilon_{0}$ and $w$ in phase-field equation as follows:

$$
\begin{gathered}
\varepsilon_{0}=\sqrt{2.73 \lambda_{0} \sigma_{0}} \\
w=6.6 \frac{\sigma_{0}}{\lambda_{0}} \\
M_{0}=\frac{T_{\mathrm{m}} \mu_{0}^{\mathrm{k}}}{2.73 \Delta H \hat{\lambda}_{0}}
\end{gathered}
$$

The numerics in Eqs. (6)-(11) are different from those in Ref. 4), which is due to the difference in defining the interface thickness. In this study, the interface thickness was defined by the distance through which $\phi$ varies from 0.1 to 0.9 . By assuming $\delta_{M}=0$ and $\delta_{\varepsilon}=0.025$, same anisotropy was applied to $2 \lambda(\theta), \sigma(\theta)$ and $\mu^{\mathbf{k}}(\theta)$. Also we used $\sigma_{0}=0.37 \mathrm{~J} / \mathrm{m}^{2}$ and $\mu_{0}^{\mathrm{k}}=2 \mathrm{~m} / \mathrm{s} \mathrm{K}$ for pure nickel melt ${ }^{91}$ and $2 \lambda_{0}=8 \times 10^{-8} \mathrm{~m}$ for numerical convenience. With $T_{\mathrm{m}}=1728 \mathrm{~K}$ and $\Delta H=2.35 \times 10^{9} \mathrm{~J} / \mathrm{m}^{3}$, we obtained $\varepsilon_{0}=2.01 \times 10^{-4}(\mathrm{~J} / \mathrm{m})^{1 / 2}, w=0.61 \times 10^{8} \mathrm{~J} / \mathrm{m}^{3}$ and $M_{0}=13.47 \mathrm{~m}^{3} / \mathrm{sJ}$. The other material parameters in Eq. (1) are $D=1.55 \times 10^{-5} \mathrm{~m}^{2} / \mathrm{s}$ and $c_{\mathrm{p}}=5.42 \times 10^{6}$ $\mathrm{J} / \mathrm{m}^{3} \mathrm{~K}$.

\section{Numerical Method}

To solve Eqs. (1) and (2), in basics we will follow the explicit finite difference method (FDM). From the preliminary computations in $200 \times 200$ mesh system with the parameters determined in previous section, a dendrite with fully developed secondary arms could not be obtained due to the small computational domain size. In order to obtain a large dendrite with several dozens of side branches the number of grid of several million are required. Therefore the computation in such a large system is restricted not only by computational efficiency, but also memory size. Here we developed a numerical technique the computational efficiency and reducing the memory size. The method is based on following three points.

- In explicit FDM, there exist stability conditions for convergence, which may be written as $\Delta t<\Delta x^{2} / 4 D$ and $\Delta t<\Delta x^{2} /\left(4 M \varepsilon^{2}\right)$. (The exact condition for convergence may be different from these conditions due to 
the non-linear terms in Eqs. (1) and (2). Direct numerical tests can show that the non-linear effect on the computational stability conditions is negligible.) The parameters determined in previous section yields $M \varepsilon^{2}=$ $5.44 \times 10^{-7} \mathrm{~m}^{2} / \mathrm{s}$, which is much smaller than the thermal diffusivity $\left(D=1.55 \times 10^{-5} \mathrm{~m}^{2} / \mathrm{s}\right)$. The time step in computation thus is limited by the thermal diffusivity, which appears as the main obstacle to the computational efficiency. In this study we circumvented this limitation by using a double-grid system: one (grid A) with a large grid size for diffusion Eq. (1) and the other (grid B) with a small grid size for the phase-field Eq. (2), which enable us to use a large time step in computation.

- If the double-grid system for the whole space is used to increase only the time step, we need a huge number of grids for phase field. However the phase field varies steeply only around the interface, and it is constant in both liquid $(\phi=0)$ and solid $(\phi=1)$ outside of the interfacial region. Therefore we can use the grid B with small grid size only around the interface and the calculation of the phase field change may be performed only on those grids, which decreases the required memory space significantly. In this case the interface position should be tracked and the grids for phase field calculation should be found at every time step, which may be regarded as an adaptive grid method. Even though the tracking of the interface at every time step spends some extra-calculation time, it decreases significantly the required memory size and also enhances the computational efficiency.

- When the Peclet number $(V R / 2 D$, where $V$ and $R$ are the dendrite tip velocity and tip radius, respectively) is large, which is just the case in this study, the thermal boundary layer does not reach far from the dendrite tip and the liquid temperature far from the dendrite tip remains constant with the given initial undercooling. Therefore the computational domain for the thermal field may be confined to the thermal boundary layer. In this case, we can save the calculation time for thermal field, especially at the initial stage of solidification.

Based on above points, we developed a numerical scheme with moderate memory space and enhanced computational efficiency. The detailed procedures are as follows.

1) The system space is divided into a uniform square grids of $I_{\mathrm{m}} \times J_{\mathrm{m}}$ with a grid spacing $\Delta X=\Delta Y$ for thermal field calculation. The temperature field $T(I, J)$ and the phase field $P(I, J)$ at each $(I, J)$ grid point are known at the previous time step.

2) For each grid $(I, J)$, an integer value $N(I, J)$ which describes the phase state of the grid is assigned: $N(I, J)=$ -1 for $P(I, J)<0.001$, indicating the grid in liquid state and $N(I, J)=0$ for $P(I, J)>0.999$, indicating solid state. For all grids with $0.001<P(I, J)<0.999$ and their nearest-neighbor grids, positive integers are assigned to $N(I, J)$ in serial manner from 1 to $N_{\mathrm{m}}$, which we define as the interfacial region. $N_{\mathrm{m}}$ is the total number of grids in the interfacial region.

3) Each grid with a positive integer number $N(I, J)$ at the interfacial region is divided into a uniform square sub-grids of $i_{\mathrm{m}} \times j_{\mathrm{m}}\left(i_{\mathrm{m}}=j_{\mathrm{m}}\right)$ with a grid size $\Delta x=\Delta y=$
$\Delta X / i_{\mathrm{m}}$. At current time step, the phase field $p(N(I, J), i, j)$ only on the sub-grids is calculated from previous values, by the explicit finite difference form of Eq. (2).

4) The average phase field $P(I, J)$ on the $(I, J)$ grid point at the interfacial region is calculated by

$$
P(I, J)=\frac{1}{i_{\mathrm{m}}+j_{\mathrm{m}}} \sum_{i, j} p(N(I, J), i, j)
$$

whereas we impose $P(I, J)=0$ in the full liquid region and $P(I, J)=1$ in the full solid region.

5) The thermal boundary layer is defined as the region with $T(I, J)>T_{\mathrm{i}}+0.1$, where $T_{\mathrm{i}}$ is a given initial undercooled temperature. A square region enveloping the thermal boundary layer around the dendrite tip is found. Note that the grids outside of the boundary layer can be also included in the square region. Whether the condition $T(I, J)>T_{\mathrm{i}}+0.1$, on grid $(I, J)$ in the square region, is satisfied or not is checked in sequence. Whenever the condition is satisfied, new temperature $T(I, J)$ at current time step is calculated from the explicit FDM form of Eq. (1), where the term $\partial \phi / \partial t$ in Eq. (1) can be calculated from the $P(I, J)$ values in previous and current time steps.

The parameters used for the representative calculation are as follows: $I_{\mathrm{m}}=J_{\mathrm{m}}=1000$ and $\Delta X=\Delta Y=6 \times 10^{-8} \mathrm{~m}$ in the grid system for temperature-field calculation and $i_{\mathrm{m}}=j_{\mathrm{m}}=3, \Delta x=\Delta y=2 \times 10^{-8} \mathrm{~m}$ in the grid system for phase-field calculation. Therefore the interface thickness $\left(2 \lambda_{0}=8 \times 10^{-8} \mathrm{~m}\right)$ corresponds to four grid spacing and the whole system size is $X=Y=60 \mu \mathrm{m}$. The initial undercooling of the nickel melt was $260 \mathrm{~K}$, corresponding to $0.6 \Delta H / c_{\mathrm{p}}$. The solidification started after changing the phase field of a seed (a small square region at the top-left corner of the system) from zero to one. We choose $\theta_{0}=\pi / 4$ in Eqs. (4) and (5). Therefore both the interface energy and the interface kinetics coefficient are maximum at the diagonal direction of a grid and, resulting in preferential dendritic growth into the direction. All the wall boundaries of the system are assumed to be adiabatic to both the thermal and phase fields. The side branching into the secondary arms around the dendrite tip is known to be possible only under existence of noise source. Therefore we added a random noise to the thermodynamic driving force (the final term in Eq. (2)), by the same way as in Warren and Boettinger. ${ }^{9)}$ The noise amplitude factor was 0.025 . The computation was performed on a personal computer with a Pentium Pro ${ }^{\circledR}$ CPU and $64 \mathrm{MB}$ RAM. The run time depends on the growth stage and will be shown in next section.

\section{Results and Discussion}

\subsection{Calculated Dendritic Pattern}

Figure 1 shows a caculated dendritic pattern in a square box $\left(I_{\mathrm{m}}=J_{\mathrm{m}}=1000, i_{\mathrm{m}}=j_{\mathrm{m}}=3\right)$, where the dendrite is growing from a seed at the top-left corner toward the bottom-right corner of the box. The run time for the calculation on the personal computer was about $72 \mathrm{~h}$. As well-known from experiments, theoretical or numerical works, the dendrite grows with a steady-state parabolic tip into the direction of the maximum interface en- 


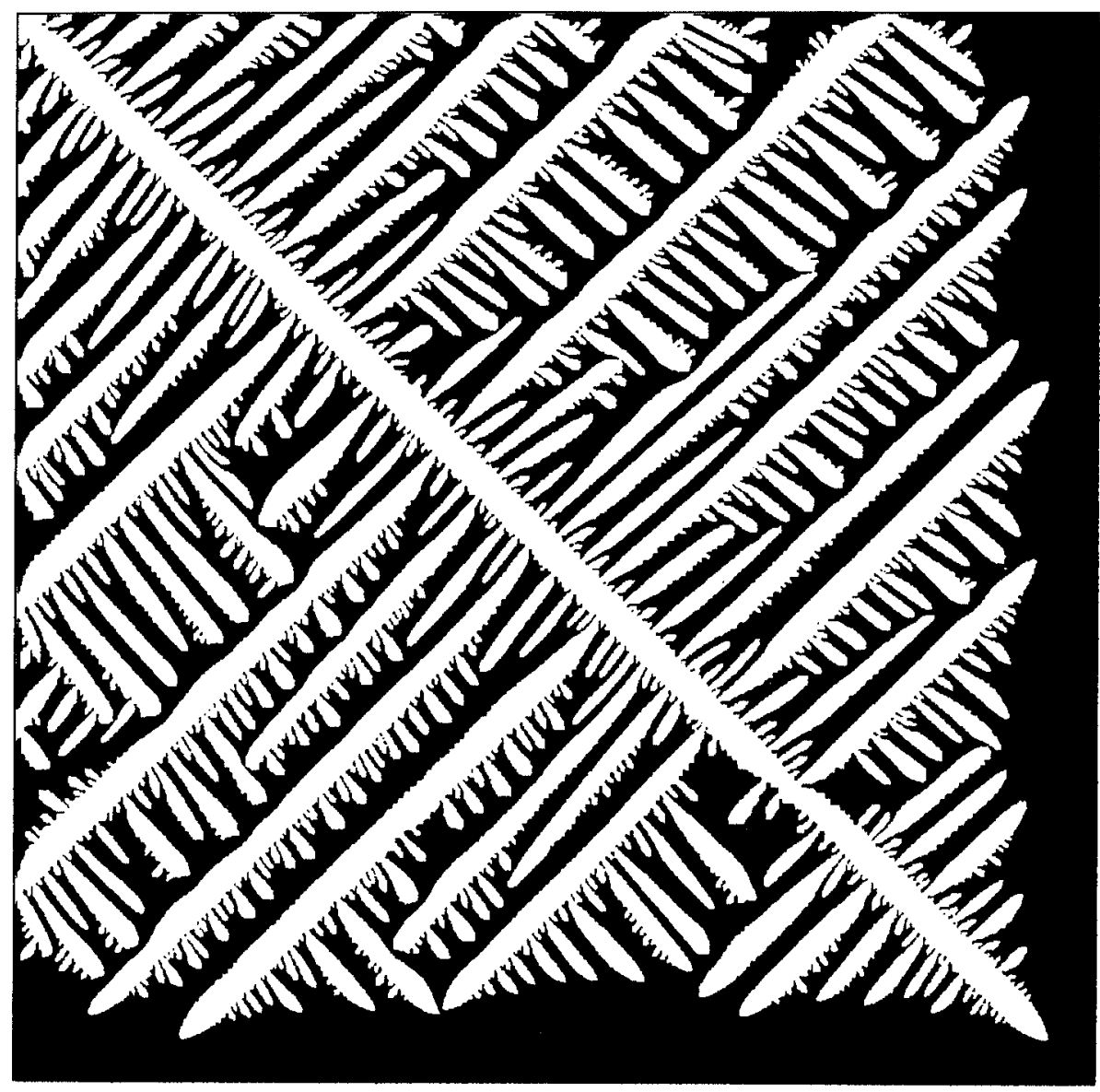

Fig. 1. A dendritic pattern calculated in a personal computer with a double grid method $\left(I_{\mathrm{m}}=J_{\mathrm{m}}=1000, i_{\mathrm{m}}=j_{\mathrm{m}}=3\right)$, where the dendrite started to grow from a seed at the top-left comer toward the bottom-right corner of the box.

ergy and interface kinetics coefficient. Note that even the tertiary arms as well as the secondary arms have grown enough form side branches.

The secondary arm spacing in Fig. 1 increases with the distance behind the primary dendrite tip. It can be cleary seen in this figure that the coarsening procedure of the secondary arms occurs by two successive stages; first, near the primary arm, the small secondary arms compete with each other and some overgrown secondary arms survive. Second, the competition between the survived arms is rather weak due to large spacing and can grow until further growth are screened by the tertiary arms. Only a few secondary arm can outgrow the neighboring tertiary arms.

One of the interesting phenomenon is the asymmetry in the side branching found in the secondary and tertiary arms. The side branching occurs only at one side of the arms, which has been observed in experiments on dendritic growth in undercooled melts. ${ }^{17)}$ The asymmetry seems to be related with the thermal field distribution. The side branching prefers to the direction to release the latent heat. The other interesting phenomena is that we can not see any side branches in some rather long secondary arms. The side-branching in secondary arm, that is, tertiary branching, appears to be dependent on the liquid-layer thickness between secondary arms, rather than the length of the secondary arm. Also there seems to be a critical thickness of the liquid-layer for the ter- tiary branching, which looks very close to the width of the primary arm. Whether the same criterion for tertiary branching in real experiments on pure melts can operates or not is unclear, because the large scale photographs showing the well-developed tertiary branches in a thermal dendrite has not been reported from out knowledge.

\subsection{Computational Efficiency}

In order to test the computational efficiency of the double-grid method, we compared the run time of the computer for the calculation with the double-grid method (method 1) and a single grid method (method 2). In method 2, we used a same grid system for both the thermal field and phase field. The grid system was $1200 \times$ 1200 with grid size of $2 \times 10^{-8} \mathrm{~m}$. Without any timesaving technique described in Sec. 3, however, the computation on the personal computer appeared to be impractical. In method 2, therefore, some of time-saving techniques were adopted. After finding a square box enveloping the thermal boundary layer, as described in Sec. 3, we scanned all the grids in sequence at every time step. During scanning, we calculated the new temperature only if the temperature of the grid satisfies the condition $T(I, J)>T_{\mathrm{i}}+0.1$, and also calculated the new phase field only if the sum of phase fields in four nearest-neighbors around the grid is between 0.004 and 0.996 . In method 1 , all the time-saving techniques de- 


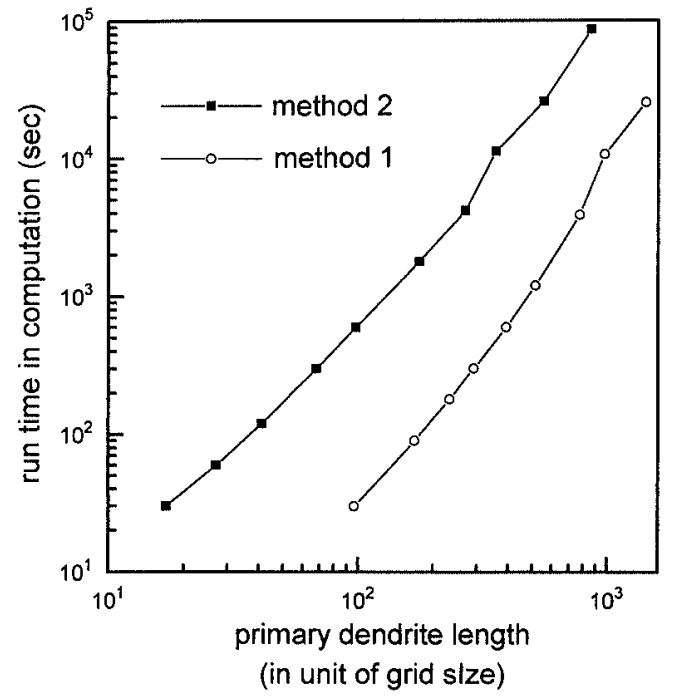

Fig. 2. Variation of run time (sec) of computer as a function of parimary dendrite length in unit of the grid size $\Delta x$ The open circles are from the double grid method (method 1) and the solid circles are from the single grid method (method 2).

scribed in Sec. 3 were adopted, and the computational parameters were as follows: $I_{\mathrm{m}}=J_{\mathrm{m}}=400$ and $\Delta X=$ $\Delta Y=6 \times 10^{-8} \mathrm{~m}$ in the grid system for temperature field and $i_{\mathrm{m}}=j_{\mathrm{m}}=3, \Delta x=\Delta y=2 \times 10^{-8} \mathrm{~m}$ in the grid system for phase field. Note that the grid size for phase field calculation and the system size were taken to be same in both two methods, whereas the time step in method 1 is larger than that in method 2 by nine times.

Figure 2 shows variation of the run time (seconds) of the computer as a function of the primary dendrite length (in unit of the grid size $\Delta x$ ), where the open and solid circles are from the method 1 and method 2, respectively. We can see that, in method 1 , the run time required to reach a given primary dendrite length is about a twentieth of that in method 2. A part of this computational efficiency is due to larger time step (nine times) in method 1 than that in method 2. The other part of the efficiency is due to using a adaptive grid for phase field calculation and smaller number of grids for thermal field calculation. With increasing the primary dendrite length, the run times in both methods increase with a power law with an exponent close two. This is a natural result because both the total interfacial area and the square box size for thermal field calculation are proportional to a square of the primary dendrite length.

Figures 3(a) and 3(b) show the dendritic patterns obtained by the method 1 and 2, respectively. The length of the primary dendrite arm is $760 \Delta x$ in Fig. 3(a) and $772 \Delta x$ in Fig. 3(b), and the run times for Figs. 3(a) and 3 (b) were 53 and $910 \mathrm{~min}$, respectively. The black lines in the figures are the contours of the solid-liquid interface drawn at time spacing of 7.68 nanosec. The steadystate growth velocity of the dendrite tip in method 1 appears to be $42.05 \mathrm{~m} / \mathrm{s}$ whereas $44.55 \mathrm{~m} / \mathrm{s}$ in method 2 . Thus the difference in the growth velocities in above two methods is only $5.62 \%$. We also measured the steady-state tip radius of the primary dendrite as follows: After rotating the dendrite by $45^{\circ}$, we found a set of the

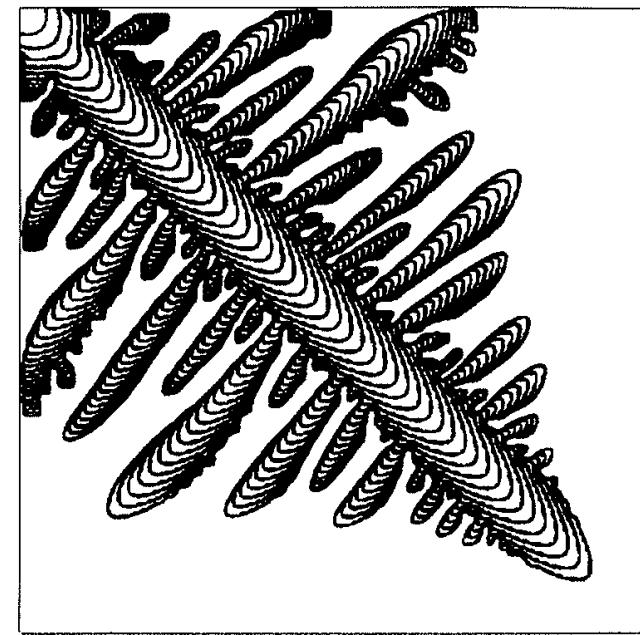

(a)

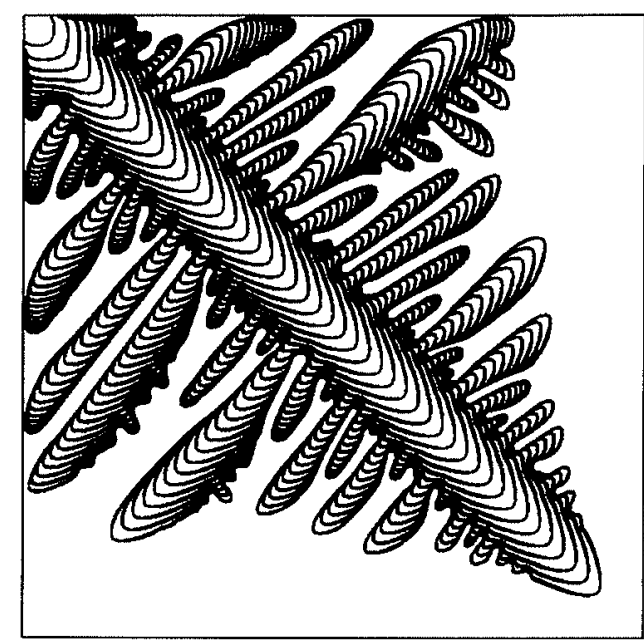

(b)

Fig. 3. Dendritic patterns obtained by (a) the method 1 and (b) the method 2. The run times of computer were (a) 53 and (b) 910 min, respectively.

interface positions $\left(x_{\mathrm{p}}, y_{\mathrm{p}}\right)$ (that is, the positions with $\phi=0.5$ ) by a four-points Lagrange interpolation for the phase-field values around the tip. Within the dendrite width about the tip radius, the interface positions were well-fitted with a parabolic curve, $y_{\mathrm{p}}=a x_{\mathrm{p}}^{2}+b$. The tip radius was calculated as $1 / 2 a$. The resulting tip radii were $189 \mathrm{~nm}$ in method 1 and $179 \mathrm{~nm}$ in method 2, from which we see the difference is only $5.59 \%$. Thus the growth procedure of the dendrite in the present doublegrid method is very close to that in the conventional single-grid method. Note that the width of the primary dendrite arm in method 1 is smaller than that in method 2 , by about $15 \%$, whereas the measured tip radius in method 1 is larger than that in method 2. This means that the side-branching instability in method 1 is stronger than in method 2. As reported frequently in experiment, ${ }^{18)}$ dendritic growth theory ${ }^{19)}$ and phase-field modeling, ${ }^{9,20)}$ the initiation of the side branching instability around the dendrite tip is very sensitive to the noise strength, whereas the tip radius and the tip velocity are nearly independent of the noise as long as the strength is not so high. The origin of difference in side-branching 
instability in both methods is unclear at this stage, even though it may be due to the larger grid size for calculation of thermal field in the method 1. Considering a great improvement in computational efficiency, however, the slight differences in dendritic patterns can not be regarded as a critical obstacle in the double-grid method. This method can be a very useful tool for qualitative description of many interface-related phenomena.

\section{Conclusion}

In this study we presented a double-grid method for efficient computation of complex dendritic pattern evolving in a large scale solidifying system of pure undercooled melts. The method, which is based on large difference in phase-field diffusivity and thermal diffusivity in pure materials, enables us to use a large time step. The phase field was calculated adaptively only on the grids within the interfacial region and also the thermal field was calculated only within the thermal boundary layer. The computation showed that the complex dendritic patterns with well-developed tertiary arms can be obtained even on a personal computer with a moderate memory space. The calculated dendritic pattern showed similar microstructure observed in previous experiments. Coarsening procedure of the secondary arms occurs by two successive stages; the strong competition between fine secondary arms at initial stage and the competition between secondary arms and tertiary arms at later stage. Also the tertiary branching from secondary arm appeared to be dependent of the liquid-layer thickness between secondary arms, rather than the length of the secondary arm.

\section{REFERENCES}

1) J. S. Langer: Directions in Condensed Matter, World Scientific, Singapore, (1986), 164.

2) G. Caginalp: Phys. Rev. A, 39 (1989), 5887.

3) R. Kobayashi: Physica D, 63 (1993), 410.

4) G. B. McFadden, A. A. Wheeler, R. J. Braun, S. R. Coriell and R. F. Sekerka: Phys. Rev. E, 48 (1993), 2016.

5) A. A. Wheeler, B. T. Murray and R. J. Schaefer: Physica D, 66 (1993), 243.

6) S.-L. Wang and R. F. Sekerka: Phys. Rev. E, 53 (1996), 3760.

7) A. A. Wheeler, W. J. Boettinger and G. B. McFadden: Phys. Rev. A, 45 (1992), 7424.

8) G. Caginalp and W. Xie: Phys. Rev. E, 48 (1993), 1897.

9) J.A. Warren and W. J. Boettinger: Acta Metall. Mater., 43 (1995), 689.

10) A. A. Wheeler, G. B. McFadden and J. B. Boettinger: Proc. R. Soc. Lond. A, 452 (1996), 495.

11) A. Karma and W. J. Rappel: Phys. Rev. E, 53 (1996), R3017.

12) A. Karma and W. J. Rappel: J. Cryst. Growth, 174 (1997), 54.

13) A. Karma and W. J. Rappel: Phys. Rev. E, 57 (1998), 4323.

14) S. G. Kim, W. T. Kim and T. Suzuki: Phys. Rev. E, 58 (1998), 3316.

15) R. J: Braun and B. T. Murray: J. Cryst. Growth, 174 (1997), 41.

16) N. Provatas, N. Goldenfeld and J. A. Dantzig: Modeling of Casting, Welding and Advanced Solidification Processes VIII, ed. by B. G. Thomas and C. Beckermann, The Minerals, Metals \& Materials Society, (1998), 533.

17) M. E. Glicksman and S. P. Marsh: Handbook of Crystal Growth I, Part B, ed. by D. J. T. Hurle, North-Holland, Amsterdam, (1993), 1078.

18) Ph. Bouissou, A. Chiffaudel, A. Perrin and P. Tabeling: Europhys. Lett, 13 (1990), 89.

19) J. S. Langer: Phys. Rev. A, 36 (1987), 3350.

20) B. T. Murrary, A. A. Wheeler and M. E. Glicksman: J. Cryst. Growth, 47 (1995), 386. 